\section{Management pathway for emergency department patients in the setting of the opioid epidemic and emergency department overcrowding}

\author{
Kiersten Gurley ${ }^{1}$, Churchill Onyeii ${ }^{2}$, Jonathan Burstein ${ }^{1,2 *}$, \\ Shamai Grossman ${ }^{1,2 *}$ \\ 'Department of Emergency Medicine, Harvard Medical School, Boston, MA, USA \\ ${ }^{2}$ Department of Emergency Medicine, Beth Israel Deaconess Medical Center, Boston, MA, USA
}

Objective The United States is currently in the midst of a major opioid addiction epidemic, of which the primary drivers are a sharp increase in prescription opioid pain medications, their misuse, and the inordinate illicit use of opioids. Declared a national health emergency, the opioid crisis puts enormous pressure on various systems, including increasing overcrowding in emergency departments (EDs) and forced changes in prescribing practices. We are piloting a newlydeveloped ED opiate pathway to streamline ED care for patients who frequently present at the ED for chronic pain management or other recurrent pain-causing medical problems.

Methods Patients at risk of possible opioid addiction are identified and their records are reviewed. If there is no narcotics agreement in place, the ED care team contacts the primary care physician and any other service providers involved in the patient's care to create a comprehensive pain management program.

Results Our pathway is simple and geared toward streamlining and improving care for patients with opioid addiction and misuse. We looked at seven patients in this pilot study with mixed results regarding decreasing future ED visits.

Conclusion This strategy may both limit opioid usage and abuse as well as limit ED visits and overcrowding by streamlining ED care for patients who frequently present for chronic pain management or other recurrent medical problems.

Keywords Opioid-related disorders; Crowding; Opioid epidemic; Public health; Critical pathways
elSSN: 2383-4625

Received: 23 July 2019

Revised: 22 September 2019

Accepted: 24 September 2019

Correspondence to: Kiersten Gurley Department of Emergency Medicine, Harvard Medical School, One Deaconess Rd, 2nd Floor Rosenberg Building, Boston, MA 02215, USA

E-mail: kgurley@bidmc.harvard.edu ORCID

https://orcid.org/0000-0002-1671-0571

*These two authors contributed equally to this work as senior author.

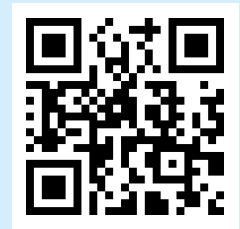

How to cite this article:

Gurley K, Onyeii C, Burstein J, Grossman S. Management pathway for emergency department patients in the setting of the opioid epidemic and emergency department overcrowding. Clin Exp Emerg Med 2020;7(2):131-135.

This is an Open Access article distributed under the terms of the Creative Commons Attribution Non-Commercial License (https:// creativecommons.org/licenses/by-nc/4.0/). 


Wapsule
Wummary is already known
The United States is currently in the midst of an opioid epidemic driven by a sharp increase in prescription opioid pain
medications, their misuse, and the inordinate illicit use of opioids. Declared a national health emergency, the opioid cri-
sis is now a top national priority and has put enormous burdens on a variety of systems, including health care and over-
crowded emergency departments (EDs), and their care and prescribing.
What is new in the current study
We have developed an ED pathway whose objective is to streamline ED care in patients who frequently present to the
ED for chronic pain management or other recurrent pain-causing medical problems. The goal is to both limit narcotic
usage and abuse as well as limit ED visits and overcrowding by streamlining ED care in patients who frequently present
to the ED for chronic pain management or other recurrent medical problems.

\section{INTRODUCTION}

The United States is currently in the midst of a major opioid addiction epidemic. The primary drivers of this epidemic are a sharp increase in the prescription of opioid pain medications and their misuse. ${ }^{1-5}$ Recent data showed that there are currently 20 million narcotic addicts, with more than 33,000 opioid-related deaths in the United States in 2016, and a measurable decrease in life expectancy of opioid users. ${ }^{6}$ The opioid crisis has been declared a national health emergency and has put enormous pressure on an already overburdened health care system, contributing to the overcrowding in emergency departments (EDs), among other factors. $^{7-9}$

According to the Centers for Disease Control and Prevention, $26 \%$ of patients using prescribed opioids become opioid-dependent, while one in 550 patients receiving chronic opioid treatment dies from opioid-related causes. ${ }^{9}$ EDs contribute to this crisis by prescribing large numbers of opioids, making them a primary source of opioid over-prescription. ${ }^{7,8,10}$ Opioid overdose has been named the eighth leading cause of mortality within the first week after an ED visit.' ED physicians face the challenge of balancing patient risk against patient discomfort daily when prescribing opioid painkillers in the absence of relevant prescribing tools and guidelines. ${ }^{111,12}$ Clinicians are forced to determine the safest treatment options, having to decide which patients are best suited for opioid treatment. ${ }^{13}$ A recent study grouped emergency physicians as either high prescribers (opioids prescribed to 25\% of ED patients) or low prescribers (prescribed to 7\% of patients), with ED patients who saw a high-prescribing physician being three times more likely to receive an opioid. ${ }^{14}$

As the opioid epidemic has escalated markedly in recent years, EDs-despite worsening overcrowding-have moved to the forefront of assisting those affected by the epidemic, caring for tens of thousands of overdose victims across all demographic groups. ${ }^{1,2,7}$ In contrast with inpatient wards and outpatient clinics, ED decisions regarding opioid management are made under intense time pressure, limiting ED physicians' ability to make complex treatment decisions regarding opioid use. ${ }^{14,15}$ Compounding this problem, physicians in an outpatient setting are becoming more restrictive in prescribing opioids, meaning that patients present at EDs for pain control and the opioid prescriptions that they would have obtained as outpatients.

Combating the opioid epidemic requires a reassessment of how clinicians provide care to addicts and overdose patients, how they view and prescribe opioids, how they provide patients with treatment resources, and how they collaborate and coordinate with other stakeholders. ${ }^{4,13}$ To address this issue, we have developed a pathway to streamline ED care for patients who frequently present at EDs for chronic pain management or other recurrent paincausing medical problems. The goal is to limit both opioid usage and abuse as well as ED visits and overcrowding.

\section{METHODS}

Patients at risk of possible opioid addiction or with frequent ED visits for opioid prescriptions are identified via referrals by quality assurance flags originating from treating ED physicians, nurses, social workers, or other ED consultative services such as psychiatry, surgical specialists or subspecialist services, or from personal knowledge. We then review the patients' integrated electronic medical records to see if there is an existing narcotics agreement in place with their primary care physician (PCP). If no narcotics agreement exists, after obtaining informed consent from the patient, we contact the PCP to ask if they are interested in creating an ED pain treatment guideline for their patient (Fig. 1). This communication would usually include the obtained data, such as "your 


\section{ED PAIN TREATMIENT GUIDELTNE}

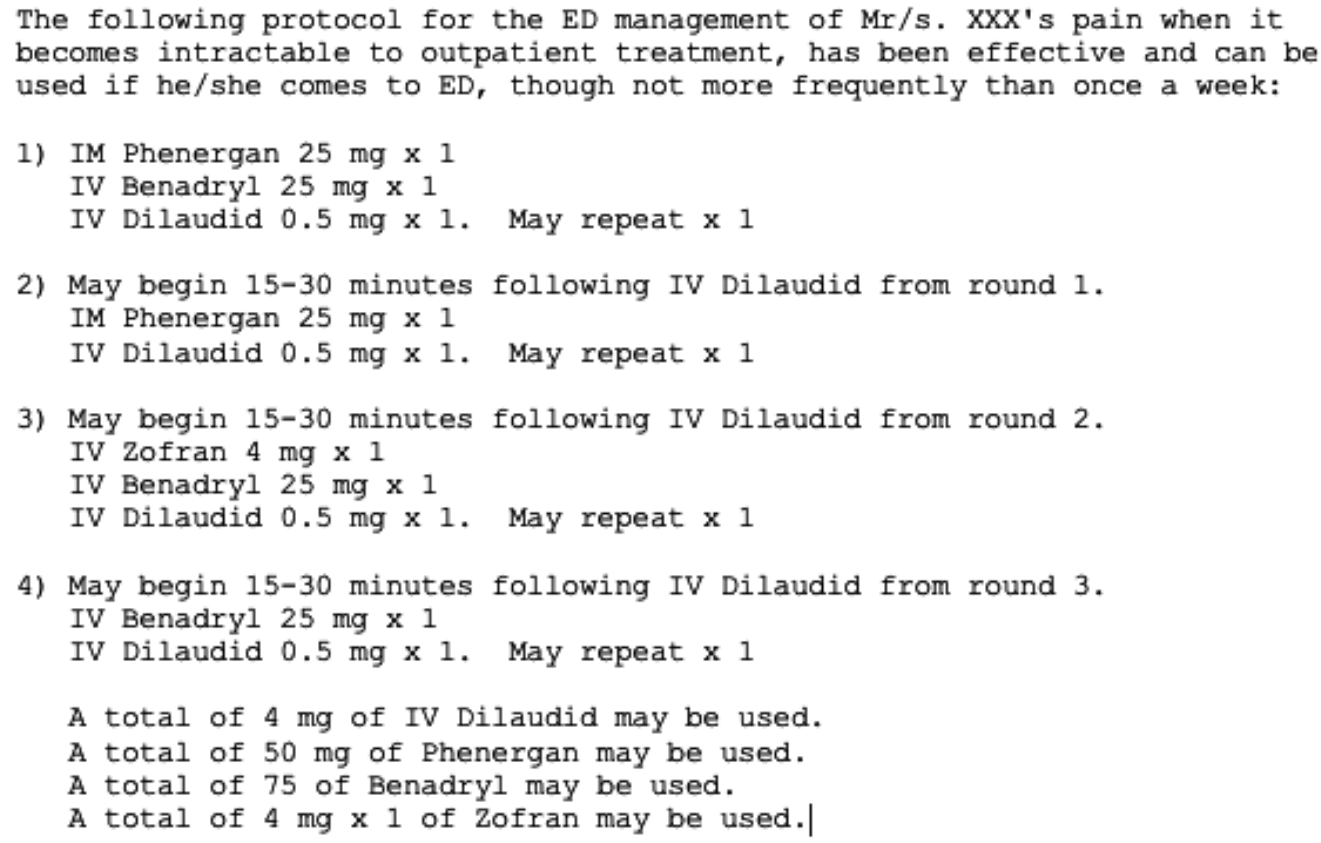

Fig. 1. Sample pain treatment protocol that can be sent to primary care physician. ED, emergency department; IM, intramuscular; IV, intravenous.

patient had 17 visits to the ED in 2017, of which 16 were to treat a headache for which they wanted hydrocodone."

If another service is involved-such as neurology for migraine or the pain management service-we contact both the specialty service and the PCP and ask if either one is interested in creating a narcotics notification and helping to formulate an ED pain treatment guideline.

If the PCP or specialist caregiver is interested, we send them sample protocols from other patients with the patient identifiers removed (Fig. 1). A typical note will read: "When patient ' $x$ ' presents, they generally benefit from IV fluid, IV diphenhydramine, and two rounds of hydromorphone $1 \mathrm{mg}$ IV." Based on these protocols, the PCP can craft a similar but individualized protocol for the patient in question and post it in a note on the electronic medical record. We rely on the PCP or other notification author to discuss it with the patient. Joint meetings and input from social work or psychiatric teams can be arranged to assist in crafting these protocols. The ED quality assurance team coordinates these meetings.

Once we receive a notification from the caregiver that the protocol has been posted, we will set a notification in the ED patient care dashboard, so the flag will automatically appear in the lefthand column of the patient's electronic record whenever the patient presents at the ED.

If the PCP or specialist caregiver is not interested in participat- ing, we do not impose a care plan independently. In such cases, particularly if the issue is egregious-such as weekly visits for IV opioids-we rather post a notification such as, "Please review recent ED visits for patterns of care." We do this rarely, but in cases when we have done so, we have emailed the PCP that the notification has been set. To date, we have not had disagreements concerning the posting of these notifications regarding patients from their respective PCPs. Given this was a quality assurance project we received exemption from our institutional review board and no consent was needed.

\section{RESULTS}

Our pathway is simple and geared toward streamlining and improving care for patients with opioid addiction and misuse. This pilot pathway has been attempted with seven patients. In two cases (29\%), the PCP declined involvement in the pathway and in five cases (71\%), the PCP agreed. In one of the five patients, there was a notable reduction (43\%) in ED visits from the previous year (32 visits to 18). In one other patient there was a modest $20 \%$ reduction in ED visits; however, in two other patients, there was an increase in ED visits (20\% and 25\% respectively). In the fifth patient, there was no change in number of ED visits (Table 1). We have started using a similar process for other patients with frequent ED visits-such as recurrent non-physiological dependency 
Table 1. Descriptive data regarding five enrolled patients

\begin{tabular}{lccl}
\hline Patient no. & $\begin{array}{c}\text { No. of visits } \\
\text { pre-guideline }\end{array}$ & $\begin{array}{c}\text { No. of visits } \\
\text { post-guideline }\end{array}$ & Rate of change \\
\hline 1 & 32 & 18 & $43 \%$ reduction \\
2 & 5 & 6 & $20 \%$ increase \\
3 & 12 & 10 & $20 \%$ reduction \\
4 & 26 & 26 & No change \\
5 & 4 & 5 & $25 \%$ increase \\
\hline
\end{tabular}

syndromes-with the goal of coordinating and improving overall patient care while limiting inappropriate use of the ED and the resultant ED overcrowding in this high-risk patient population.

\section{DISCUSSION}

To address the opioid crisis, providers need to shift from viewing addiction as a moral failure to rather viewing the problem as a treatable chronic illness. ${ }^{1-15} \mathrm{EDs}$, by helping to prevent inappropriate opioid use and identifying as well as treating those affected by the opioid crisis, can play an invaluable role, both in helping their patients and preserving ED capacity for acute illnesses. . $^{1016-18}$ Hospital leadership and health systems must fully support clinicians in providing patients with resources and treatments and in addressing the system-based issues driving ED overcrowding. ${ }^{18}$ In synergy, the government needs to increase its efforts to reduce the public's exposure to opioids, improve access to safe and effective addiction treatment, and help ensure the effective use of EDs through appropriate legislation. ${ }^{19} \mathrm{~A}$ novel adjunct has been the use of 'navigators.' These are ED staff allocated to follow up with the patients at home after they have been discharged from the ED to ensure that they receive the appropriate treatments and support. ${ }^{15}$

Standardization initiatives in the ED, such as opioid prescribing guidelines, have been suggested to reduce both the volume of opioid prescriptions as well as number of new addictions by decreasing opioid prescriptions. ${ }^{17}$ To address the variability in prescribing practices, hospitals and health care systems should ensure that clinicians and staff have the appropriate resources readily available, including prescribing guidelines, prescriber feedback, and patient education material. ${ }^{13,15}$

Future pathway development and goals, as the use of this pathway becomes more widespread, include the measurement of ED length of stay, finding variables that identify success, and performing specific outcome analyses. We plan to observe the postimplementation metrics of care pathways with the goal of ultimately discerning whether the pathway implementation has resulted in reductions in number of ED visits for individual patients as well ED visits in general for patients needing opioid-related care.

Although this is as a pilot study, we have anecdotally seen that our pathway could limit both opioid abuse and-at least in one case-limit the frequency of ED visits. It is clear that robust data are critical to confirm scientific validity, and we plan to continue this study to establish its utility. Additionally, we have tailored our pathway to our in-house ED dashboard notification system. To use this system in other institutions, the logistics and electronic medical record system would have to be tailored appropriately.

Nevertheless, we believe that the principles of this notification system and the pathway in general remain the same, regardless of the individual hospital or patient care system. This pathway could be utilized in a paper format as well.

To summarize, creating an opioid management pathway may streamline ED care for patients who frequently present at the ED for chronic pain management or other recurrent medical problems. This strategy may both limit opioid usage and abuse as well as ED visits and overcrowding.

\section{CONFLICT OF INTEREST}

No potential conflict of interest relevant to this article was reported.

\section{REFERENCES}

1. Murthy VH. Ending the opioid epidemic: a call to action. N Engl J Med 2016;375:2413-5.

2. US Department of Health and Human Services. What is the U.S opioid epidemic? [Internet]. Washington, DC: US Department of Health and Human Services [cited 2018 Apr 16]. Available from: http://www.hhs.gov/opioids/about-the-epidemic.

3. National Center for Injury Prevention and Control. Opioid painkiller prescribing: where you live makes a difference [Internet]. Atlanta, GA: Centers for Disease Control and Prevention; 2014 [cited 2018 Apr 16]. Available from: https://www. cdc.gov/vitalsigns/opioid-prescribing/index.html.

4. Graham K. Op-Ed: inaction on the opioid crisis killing our people and our economy [Internet]. Ontario: Digital Journal; 2017 Sep 21 [cited 2018 Jul 9]. Available from: http://www.digitaljournal.com/life/health/op-ed-inaction-on-the-opioid-crisisis-killing-people-and-our-economy/article/503032.

5. Wilson R. Opioid crisis is getting worse [Internet]. Washington, DC: The Hill; 2018 Mar 8 [cited 2018 Apr 15]. Available from: https://thehill.com/homenews/state-watch/377414new-data-shows-opioid-crisis-is-just-getting-worse.

6. Stein R. Life expectancy drops again as opioid deaths surge in 
U.S. [Internet]. Washington, DC: National Public Radio; 2017 Dec 21 [cited 2018 Apr 16]. Available from: https://www.npr. org/sections/health-shots/2017/12/21/572080314/life-expectancy-drops-again-as-opioid-deaths-surge-in-u-s.

7. Goodchild B, Gray S. Battling opioid dependence in the emergency department: emergency medicine professor says EDs can help prevent, identify and treat narcotics abuse [Internet]. Worcester, MA: University of Massachusetts Medical School; 2015 Mar 12 [cited 2018 Apr 16]. Available from: https:// www.umassmed.edu/news/news-archives/2015/03/battlingopioid-dependence-in-the-emergency-department/.

8. Oaklander M. How doctors are fueling the opioid epidemic [Internet]. New York, NY: Time USA; 2017 Feb 18 [cited 2018 Apr 15]. Available from: https://time.com/4675325/doctorsopioid-epidemic/.

9. Hoban B. The far-reaching effects of the US opioid crisis [Internet]. Washington, DC: Brookings; 2017 Oct 25 [cited 2018 Apr 16]. Available from: http://www.brookings.edu/bog/brookings-now/2017/10/25/the-far-reaching-effects-of-the-USopioid-crisis/.

10. Serafini M. The physicians' quandry with opioids: pain versus addiction. NEJM Catal 2018 Apr 26. https://catalyst.nejm.org/ doi/full/10.1056/CAT.18.0195.

11. Zezima K. As the opioid epidemic rages, the fight against addiction moves to an Ohio court room [Internet]. Washington, DC: The Washington Post; 2018 Apr 8 [cited 2018 Apr 16]. Available from: https://www.washingtonpost.com/national/ as-the-opioid-epidemic-rages-the-fight-against-addictionmoves-to-an-ohio-courtroom/2018/04/07/97b82b84-263611e8-874b-d517e912f125_story.html.

12. Thompson D. ER visits for opioid overdose soaring: CDC [Internet]. New York, NY: WebMD; 2018 [cited 2018 Apr 16]. Avail- able from: https://www.webmd.com/drug-medication/news/ 20180306/er-visits-for-opioid-overdoses-soaring-cdc\# 1.

13. Laderman M, Martin L. Health care providers must act now to address the opioid crisis. NEJM Catal 2017 Apr 19. https:// catalyst.nejm.org/doi/full/10.1056/CAT.17.0496.

14. Barnett $M L$, Olenski $A R$, Jena $A B$. Opioid-prescribing patterns of emergency physicians and risk of long-term use. N Engl J Med 2017;376:663-73.

15. Farmer B. Patient safety in the emergency department. Emerg Med 2016;48:396-404.

16. Blatt J, Araspacochaga D. Addressing the prescription opioid crisis: advancing provider education and collaboration with all stakeholders. NEJM Catal 2017 Nov 8. https://catalyst.nejm. org/doi/full/10.1056/CAT.17.0346.

17. National Center for Injury Prevention and Control. Problem: an increase in painkiller prescribing is a key driver of the increase in prescription overdoses [Internet]. Atlanta, GA: Centers for Disease Control and Prevention; 2014 [cited 2018 Apr 15]. Available from: https://www.cdc.gov/vitalsigns/opioidprescribing/index.html\#problem.

18. Lawlor J. Maine emergency department visits for opioid overdoses jumped 34 percent in 2017 [Internet]. South Portland, ME: Portland Press Herald; 2018 Mar 7 [cited 2018 Apr 16]. Available from: https://www.pressherald.com/2018/03/07/ maine-emergency-department-visits-for-opioid-overdosesjumped-34-percent-in-2017/.

19. Vuolo L. Will declaring the opioid epidemic a national emergency lead to a public health approach or a return to the war on drugs? [Internet]. New York, NY: Center on Addiction; 2017 Oct 17 [cited 2018 Apr 16]. Available from: https://www.centeronaddiction.org/the-buzz-blog/will-declaring-opioid-epidemic-national-emergency-lead-public-health-approach-or. 